\title{
COALITIONAL ENERGY PURCHASING IN THE SMART GRID
}

\author{
Meritxell Vinyals, Filippo Bistaffa, Alessandro Farinelli \\ Department of Computer Science \\ University of Verona, Italy \\ \{meritxell.vinalssalgado,alessandro.farinelli\}@univr.it
}

\author{
Alex Rogers \\ Agents, Interaction and Complexity Group \\ School of Electronics and Computer Science \\ University of Southampton,UK \\ acr@ecs.soton.ac.uk
}

\begin{abstract}
Matching demand and supply is recognized as a crucial issue for smart grids, and ICT-based solutions are essential to deliver the infrastructure, algorithms and mechanisms for demand-supply balancing. To date, most work in this area focus on providing users with real time feedback on energy prices and consumption, or on load scheduling of home appliances for individual user consumption. In this paper, we take a complementary approach by exploiting social relationships among consumers to organise them into coalitions of Virtual Electricity Consumers (VECs) that buy electricity as a single customer in order to get a discount on electricity through collective buying. Specifically, we model our problem as a coalitional game and provide an algorithm, based on linear programming, to form VECs. The VECs formed by our algorithm are both efficient (i.e., minimizing the sum of users' payments) and stable (i.e., no user has any incentive to break away). We empirically analyse our approach using real consumption data for a set of households located in UK. Our analysis provides interesting insights into the relationship between structure and stability of VEC's and prices within the electricity market.
\end{abstract}

Index Terms - Coalition formation, virtual electricity consumer, collective buying, demand-side management

\section{INTRODUCTION}

Balancing demand and supply is a crucial issue for the electricity grid currently achieved by varying the supply-side to continuously match demand. Demand is typically met through a combination of base-load stations which are cheap but slow to vary, and more expensive peaking-plants whose outputs can readily change to follow changing demand. Although only running at peak time when there is high demand, these generators are responsible for a significant part of consumers electricity bill.

In this perspective, the vision of the Smart Grid includes demand-side peak-shaving strategies such as real-time pricing or profile's based tariffs to encourage consumption that flattens demand [1]. A flattened demand results in a more efficient grid not only with lower carbon emissions but also with lower prices for customers. ICT-based solutions are needed for the implementation of these demand side programmes in order to help consumers to buy energy in the changing markets and reduce their energy bills. Hence, several works focus on developing IT infrastructures that provide direct real time information to consumers about tariffs, consumption and load [2].

However, while promising, when each consumer independently optimizes its own consumption, the effectiveness of these solutions has a clear limit on the consumer's restrictions and comfort (e.g. a consumption peak in the non-working hours of consumers is very likely to occur). More interestingly, recently a number of organizations, such as the Big Switch ${ }^{1}$ in the UK, have captured a lot of attention by bringing consumers together in order to get a discount on electricity through collective buying (i.e. by encoring customers to harness the power of group buying).

Similarly, in this paper, we focus on the formation of coalitions among electricity consumers with near-complementary consumption restrictions. We then assume that a coalition of consumers acts in the market as a single virtual electricity consumer (VEC) with a flattened demand profile for which it can negotiate better prices. This reduced price is related to the composition of electricity market that, in most European countries includes at least two different kinds of markets: the spot and the forward electricity markets [3]. The spot markets trade short-term electricity deliveries, and typically each time slot may have a different unit price for electricity (e.g., in Italy the day-ahead market is a spot market where hourly blocks of electricity are negotiated for the next day [4]). In contrast, forward markets trade electricity contracts for long periods (e.g. month, quarter or year) with delivery and withdrawal obligations. Thus, the contract in a forward market specifies a single quantity that will be delivered at constant rate for the contract period and a single price. Customers can benefit from lower rates in the forward markets but to participate they must be sure to use all the energy they buy. Therefore a flat coalitional profile allows customers to buy more on the forward market and consequently obtain higher gains. On the other hand, if customers buy more energy on

\footnotetext{
${ }^{1}$ https://www.whichbigswitch.co.uk/
} 
the forward market suppliers can have a better estimate of future electricity demand and thus they can better optimize production.

Thus, against this background, in this paper we propose a game-theoretical model for VEC formation that finds the most efficient VECs to form (i.e., the VECs that minimize the sum of payments). Furthermore we show how to split total payment among its members so that the VECs are stable (i.e., no user or group of users has any incentive to leave and form on other VECs). Our solution is based on modelling the electricity consumer coalition formation problem as a coalitional game [5], where: (i) the set of coalitions with maximum collective value (i.e., an optimal coalition structure), has to be identified; and (ii) each coalition's value has to be distributed among its members in such a way that coalition members have no incentive to break away from the identified optimal structure. Moreover, we restrict the coalition membership using the individual customers' acquaintances in a social network to provide some form of trust among coalition members.

Thus, in more detail, this paper makes the following contributions:

- We formally define the concept of VEC along with a metric that, given an estimation of the aggregated coalition consumption, computes the total payment by optimizing the buying strategy within the electricity markets.

- We propose an algorithm that allows consumers in a social network to generate and evaluate the networkfeasible electricity coalitions.

- We use linear programming approaches to identify the most efficient VEC's and to subsequently allocate corestable payments to individual consumers (if the core is not empty). When such core-stable allocation exists the total payment of each VEC is split among its members such that the formed VECs are stable (i.e. consumers can not be better off leaving their current VEC and forming a new one).

- We evaluated our model on a real dataset based on the electricity consumption of households in the UK. The results are analyzed in terms of the structure and stability of the formed coalitions as well as in terms of the gain obtained by consumers as a result of engaging in the coalition formation process. Results show that stability of the most efficient VECs is significantly affected by the density of the social network. Moreover, the structure of the formed VECs is highly dependent on the difference between market prices; close prices between forward and spot market do not encourage the formation of coalitions, whereas, as this difference increases, larger coalitions appear (until after some limit parameter value the grand coalition always emerges).

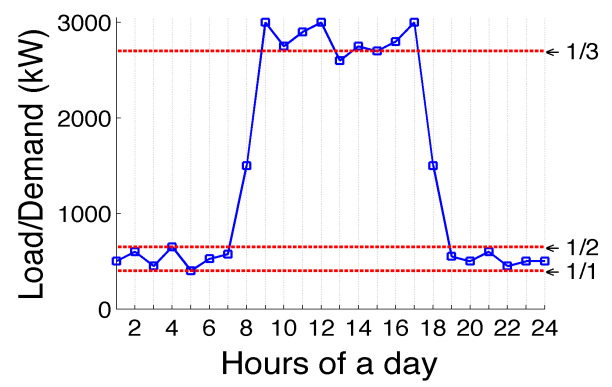

Fig. 1. A sample of a hourly load electricity profile and the different quantities to buy in the forward market given different forward over spot market prices ratios.

This paper is structured as follows. In Section 2, we describe our model for coalition formation among electricity consumers as a coalitional game. Section 3 presents our empirical evaluation, and Section 4 concludes the paper.

\section{THE MODEL}

In this section, we model the problem of group formation among electricity consumers as a coalitional game. Let $A=$ $\{1, \ldots, n\}$ be the set of electricity consumers ids, each one with its associated electricity profile. Figure 1 shows an example of an electricity profile as a graph that plots the variation in the electrical load (measured in $\mathrm{kW}$ ) versus time (measured in hours). Formally, we define the electricity profile of a consumer $i$ as a vector $e_{i}=\left\{e_{i}^{1}, \ldots, e_{i}^{N}\right\}$ where $e_{i}^{t}$ is the amount of electricity consumed at time slot $t$.

Consumers can form electricity coalitions $S \subseteq A$, where an electricity coalition $S$ stands for the set of consumers $S$ acting as a VEC in the market along with their joint consumption. We refer to the coalition composed of all consumers as grand coalition and to the coalition composed by a single individual consumer as singleton coalition. Then, the coalition formation process involves three differentiated activities: (1) determining which coalitions can be formed and the metric to evaluate them (Coalitional Value Calculation); (2) finding a coalition structure ${ }^{2}$ with maximal value (Coalition Structure Generation); and (3) dividing the payments generated by the total consumption of their electrical coalitions (Payoff Distribution). Next, we specify how we solve the three main activities that underline the coalition formation process for this particular domain.

\subsection{Coalitional Value Calculation}

In this section we formalize the coalitional value calculation, namely the generation and evaluation of coalitions, for the VEC formation. In particular, we propose that consumers use social networks to support the formation and restriction of electricity coalitions.

\footnotetext{
${ }^{2} \mathrm{~A}$ coalition structure refers to a partition of the space of consumers into coalitions.
} 
Coalition Evaluation. The first issue that arise in this context is how consumers evaluate the potential of a VEC. To this end we define a metric to evaluate coalitions by computing the total payment that coalition of consumers $S$ will incur for their aggregated demand. We represent the (expected) demand of any coalition of consumers $S$ by their joint average electricity profile $e_{S}=\left\{e_{S}^{1}, \ldots, e_{S}^{N}\right\}$ where $e_{S}^{t}=\sum_{i \in S} e_{i}^{t}$.

Now we propose a metric that optimises the buying strategy across the forward and the spot markets taken to meet the expected VEC aggregate consumption. Let $p_{F}$ be the unit electricity price in the forward market and $p_{S}$ the average unit electricity price among daily hours in the spot market ${ }^{3}$. The value of the expected payment for the coalition $S$ is given by:

$$
v(S)=\sum_{t=1}^{N} q_{S}^{t}(S) \cdot p_{S}+N \cdot q_{F}(S) \cdot p_{F}+\kappa(S)
$$

where $q_{F}(S)$ stands for the time unit amount of electricity to buy in the forward market and $q_{S}^{t}(S)$ for the amount to buy in the spot market at time slot $t$ such that satisfy:

$$
q_{S}^{t}(S)+q_{F}(S) \geq e_{S}^{t} \quad \forall t=1 \ldots n
$$

and where $\kappa(S)$ stands for a coalition management constant, a constant that depends on the size of the coalition and captures the intuition that larger coalitions are harder to manage so in case of equal value consumers prefer smaller ones. In particular, through this paper, we set this constant as $\kappa(S)=$ $(|S|-1) \cdot\left(\max _{t=1 \ldots n} q_{S}^{t}(S)-q_{F}(S)\right) /|A(G)|$.

Hence, to compute the value of a coalition in this domain consumers need to determine the forward and time-slot spot quantities such that Equation 1 is maximised (i.e. the payment regarding their joint consumption is minimized) whereas satisfying constraints in Equation 2 that guarantee that these quantities meet the coalition electricity demand. Algorithm 1 describe a procedure that allows agents to solve the abovedefined optimization problem.

ALGORITHM 1. Given as input the coalition electricity profile, $e_{S}$, and the ratio between prices among the two available markets, $\frac{p_{F}}{p_{S}}$ the algorithm returns $q_{F}(S)$ and for each time slot $t q_{S}^{t}(S)$.

1(a): Sort the coalition profile values $e_{S}$ in descending order.

1(b): Set $q_{F}(S)$ to the value at position $p_{F} / p_{S} \cdot N$ of the profile (that is the amount of energy covered at least $\frac{p_{F}}{p_{S}}$ of the time interval).

1(c): For each time slot $t=1 \ldots N$ compute the spot quantity for a time slot $t$ as the amount of demanded electricity that exceeds the forward quantity, that is $q_{S}^{t}(S)=$ $\max \left(e_{S}^{t}-q_{F}(S), 0\right)$;

Intuitively, in order for consumers in a coalition to be advantageous to buy a certain continuous electricity amount $q_{F}$ for

\footnotetext{
${ }^{3}$ Prices are negative values to denote the direction of payment
}

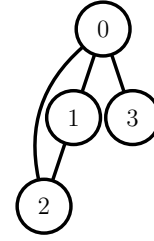

(a) $G$

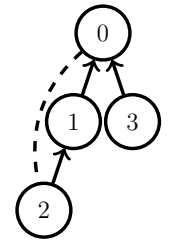

(b) $P T$

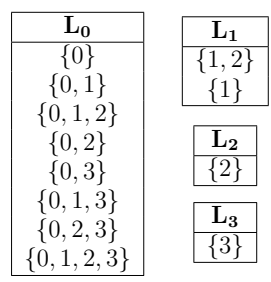

(c) $G$-restricted coalitions
Fig. 2. Example of (a) a consumer network $(G)$; (b) a pseudotree $P T$ of $G$; and (c) the set of $G$-restricted coalitions partitioned in leading coalitions per consumers.

all the period in the forward market, this continuous amount should be used at least $\frac{p_{F}}{p_{S}}$ of the time interval. Figure 1 shows how different market ratios lead to different buying strategies within electricity markets for the same coalition profile. Horizontal lines stand for different quantities to be bought in the forward market given the market ratios as labeled at the end of the line. Observe that in the particular case when there is no economical incentive to buy in the forward market $\left(p_{F}=p_{S}\right)$, the forward quantity represents the safer base load corresponding to the minimum among the hourly expected consumption (in Figure 1 the consumption at time slot 3). Notice that as the $\frac{p_{F}}{p_{S}}$ ratio decreases, VECs increase the quantity bought in the forward market, in detriment of this bought in the spot, buying larger continuous amounts even when they are not expect to used it all hours of the day. Thus, in Figure 1 if the ratio between markets is one half the amount to buy in the forward market is exactly the value of the 12th measure in magnitude (corresponding to time slot 4).

Once provided a metric that allows consumers to evaluate electricity coalitions, the second issue that consumers need to address is to decide which coalitions consider and how to enumerate them.

Coalition enumeration. We consider that each consumer looks for potential partners for its coalitions through its contacts in a social network. In this way, coalition membership is restricted to coalitions composed of friends of friends, being always somebody in the coalition responsible for the introduction of a new member. From the game perspective this implies that feasible coalitions are restricted by a graph: (i) each node of the graph represents a consumer; and (ii) a coalition $S$ can be formed iff every two consumers in $S$ are connected by some path in the subgraph induced by $S$. Let $G$ be a connected (undirected) graph with vertex set $A(G)=\{1, \ldots, n\}$ and let $E(G)$ be the set of edges among consumers. We denote the set of feasible coalitions as $F(G)$. An example of a 4-consumer network that defines a graph $G$ is given in Figure 2 (a). Tables in Figure 2 (b) list the set of feasible coalitions restricted by the graph in Figure 2 (a). Thus, consumer 3 can form an electricity coalition with 0 and $1(S=\{0,1,3\})$ but not a coalition with 1 without 0 
$(S=\{1,3\} \notin F(G))$.

Here, we observe that the problem of generating graphcoalitions can be cast to the problem of enumerating all connected induced subgraphs of a given graph and we use the state-of-the-art algorithm proposed in [6] to generate all electricity coalitions in the social network. This algorithm (for further details see [6] Section 5) (i) uses an ordering among consumers that partitions the set of graph coalitions into disjoint (leading) sets $\left\{\mathbf{L}_{i} \mid a_{i} \in A(G)\right\}$, one per consumer; and (ii) can be executed independently by each consumer $i \in$ $A(G)$ to generate its set of leading coalitions $\mathbf{L}_{i}$ (i.e.all graph coalitions in which $i$ has precedence position in the ordering among other coalition members). Instead of using a linear ordering as in [6], we propose to use the partial ordering that defines a pseudotree arrangement of the social network graph [7]. A pseudotree $P T$ of $G$ is a rooted tree with consumers $A(G)$ as nodes and the property that any two consumers that share an edge in $G$ are on the same branch in $P T$. Figure 2(b) shows a pseudotree, rooted at consumer 0 , of the cyclic graph $G$ in Figure 2(a) and Figure 2(c) shows the different sets of agents leading coalitions for the pseudotree in Figure 2(b).

\subsection{Coalition Structure Generation}

To solve the Coalition Structure Generation (CSG) problem, which is known to be NP-Hard, we use an integer programming (IP) approach (see [8], pages 38-39). Compared with other state-of-the-art CSG algorithms [9, 10], this approach has the important advantage that can restrict the search only to any feasible set of coalitions and, in particular, to the graphbased coalitions such as the ones we are interested in. The CSG problem is formulated as a binary integer programming problem containing a set of binary decision variables $x_{S} \in$ $\{0,1\}$, one per feasible coalition $S \in F(G)$. Then, solving the CSG amounts to solving the following IP:

$$
\max \sum_{S \in F(G)} v(S) \cdot x_{S}
$$

such that each consumer can join at most one coalition:

$$
\forall a_{i} \in A: \sum_{S \in F(G) \mid S \ni i} x_{S}=1
$$

where having a variable $x_{S}=1$ corresponds to coalition $\mathrm{S}$ being selected in the optimal coalition structure $C S^{*}$.

\subsection{Payoff Distribution}

Having described how coalitions are formed, we now focus on how consumers in a VEC should share the payment for their aggregated consumption. Since consumers are selfish (i.e. they are only concerned with maximizing their own payoffs) to guarantee stability we need to divide the payment of each coalition among its members in such a way that consumers have no incentive to deviate (i.e. there are no other outcomes that can make them better-off). From a game theoretic point of view, this involves to determine core-stable payments of the game.

Given the optimal coalition structure, we can compute the core-stable payments or alternatively, detects its nonexistence (i.e. the emptiness of the core) by solving a linear program (LP) ${ }^{4}$. Our aim is to find a set of negative real values that represents the consumers' payments $\rho$, one $\rho_{i} \in \rho$ for each consumer $i \in A(G)$. Finding such a stable payments then amounts to solving the following LP:

$$
\min \sum_{i \in A(G)} \rho_{i}
$$

such that there are no deviating coalitions for these payments:

$$
\forall S \in F(G): \sum_{i \in S} \rho_{i} \geq v(S)
$$

and consumers' payments are equal or lower than 0 (i.e. agents do not make a positive profit exploiting other consumers)

$$
\forall i \in A(G): \rho_{i} \leq 0
$$

If the value of the objective function of this LP yields the value of the optimal coalition structure then, the problem has a non-empty core and the values in $\rho$ define an allocation in the core. Otherwise, the problem has an empty core. It should be emphasized that the optimal coalition structure $C S^{*}$ is a given parameter which means that although this program can be solved in polynomial time it needs as input the outcome of the IP program defined in Section 2.2 which is NP-Hard.

\section{EMPIRICAL EVALUATION}

In this section we provide an empirical evaluation of the coalition formation model introduced in Section 2. The IP and LP problems defined in Sections 2.2 and 2.3 for computing the optimal coalition structure and the core-stable payments are solved using an standard, off-the-shelf optimization software (specifically CPLEX 12.3). First, we explain the details of our experimental setup in Section 3.1. Next, we analyse our empirical results in Section 3.2.

\subsection{Empirical settings}

To analyse the sensitivity of the coalition formation process with respect to the underlying network topology, we evaluate our model for two different network models, namely random and scale free networks ${ }^{5}$. Moreover, each network model is tested on three different link densities ${ }^{6}: d=1$ (low), $d=2$ (medium) and $d=3$ (high). Notice that scale free

\footnotetext{
${ }^{4}$ Linear programs can be solved in polynomial time in the number of variables and constraints.

${ }^{5}$ Results obtained in small-networks are omitted for the sake of space due to their similarity to those obtained in scale free networks.

${ }^{6}$ the density of a graph is defined as the ratio between the number of links and the number of agents in the graph $\left(\frac{|E|}{|A|}\right)$
} 


\begin{tabular}{|c|ccc|}
\hline Market $(€ / \mathrm{KWh})$ & M1 & M2 & M3 \\
\hline $\mathbf{p}_{\mathbf{F}}$ & 70 & 60 & 40 \\
\hline $\mathbf{p}_{\mathbf{S}}$ & 80 & 80 & 80 \\
\hline
\end{tabular}

Table 1. Different market conditions tested in experiments.

networks are known to capture some characteristics of social networks [11], while random networks constitute a more synthetic model for our domain. For each instance, the electricity profile of each consumer is randomly selected from a real dataset composed of monthly average electricity profiles ${ }^{7}$ characterizing the real domestic electricity consumption of 5000 households in the UK. All experiments are run using networks of 20 consumers.

Finally, as described in Section 2, the value of a coalition in our model depends on the two market prices, the price of the electricity in the forward and the spot market, we evaluated our model under three market conditions detailed in Table 1. Market M1 uses real prices of the forward and day-ahead market (that forms part of the spot electricity markets) in Italy [4] whereas M2 and M3 explore scenarios in which buying in the forward market is more beneficial.

\subsection{Results}

We evaluate our model by performing 50 simulations for each configuration detailed above. The next sections provide an analysis of our results in terms of consumers social gain and the stability and structure of the formed coalitions.

\subsubsection{Consumers social gain}

In this section we analyse the consumers' effective gain obtained by adopting the coalitional approach proposed with respect to the non-coalitional one, composed of singleton coalitions. Let $v\left(C S^{*}\right)$ be the total payment of agents in the final coalitions and $v(\{i\})$ the payment of the agent in its singleton coalition. Then, the average percent gain is assessed as $\frac{v\left(C S^{*}\right)-\sum_{a_{i} \in A} v(\{i\})}{\sum_{a_{i} \in A} v(\{i\})}$. Table 2 shows the average percent gain for 20 consumers on a random and scalefree networks in the three different market scenarios respectively.

Results show that in all configurations the coalitional percent gain increment with density (more links among agents lead to more feasible electricity coalitions among them) is not significant. Regarding different market conditions, as expected, the average percent coalitional gain is directly proportional to the economical incentive to enroll the forward market: around $0.5-0.6 \%$ in $\mathrm{M} 1,1.1-1.4 \%$ in $\mathrm{M} 2$ and $6.6-7.2 \%$ in $\mathrm{M} 3$.

\subsubsection{Economical stability of electricity coalitions}

As argued in Section 2, core-stable payments prevent selfish consumers to continually moving from coalition to coalitions leading to unstable coalition structures with possibly

\footnotetext{
${ }^{7}$ Each consumer has been monitored during December 2009, recording the power consumption every half an hour, for a total of 48 daily time slots.
}

\begin{tabular}{|l|l|c|c|c||c|c|c|}
\hline \multirow{2}{*}{ Topology } & \multirow{2}{*}{ Density } & \multicolumn{3}{|c||}{ \% Average Gain } & \multicolumn{3}{c|}{ \% Empty Core } \\
\cline { 3 - 8 } & & M1 & M2 & M3 & M1 & M2 & M3 \\
\hline \multirow{3}{*}{ Random } & Low & 0.5 & 1.1 & 6.6 & 0 & 2 & 0 \\
& Medium & 0.5 & 1.3 & 7.2 & 48 & 31 & 58 \\
& High & 0.6 & 1.3 & 7.1 & 54 & 48 & 59 \\
\hline \multirow{3}{*}{ ScaleFree } & Low & 0.6 & 1.4 & 7.2 & 0 & 0 & 0 \\
& Medium & 0.6 & 1.4 & 7 & 50 & 40 & 52 \\
& High & 0.6 & 1.4 & 7.2 & 64 & 60 & 52 \\
\hline
\end{tabular}

Table 2. Average coalitional gain and percentage of instances with empty core under different configurations.

less social value (i.e. the sum of payments across VECs is greater than in the optimal). Table 2 shows the percentage of instances for each configuration for which the core was detected as empty (i.e. core-stability does not exist). Notice that in all network topologies, the number of instances for which the core is empty increases with the density of the network. Observe that for low density networks most instances create core-stable coalitions $(98 \%-100 \%)$. These results are coherent with the well-known results that any acyclic network (which has by definition the lowest density) is guarantee to have a non-empty core [12]. As we increase the density the number of cycles also increases and results show that the probability of core emptiness is higher (i.e. a higher number of instances have no stable economical agreement among consumers). Thus, for example, in high density networks more than one half of instances resulted in non-stable coalitions.

\subsubsection{Structure of electricity coalitions}

In this section we analyse the structure of the electricity coalitions obtained in the experiments. For each configuration we plot the median of the minimum and maximum size of coalitions formed as well as the median of the number of coalitions in the optimal coalition structure. Figure 3 plots the results for networks of 20 consumers for random and scale free networks in the three explored market scenarios. We also plot the standard error of the mean as a measure of the variance in each graph. We observe that for markets M1 and M2, the median number of coalitions of the optimal coalition structure varies from around 3 to 6 coalitions for 20 agents under all configurations. Thus, market conditions M1 and M2 lead to coalitions of middle size in all network structures. In contrast, market scenario M3 leads to much larger coalitions in all configurations, and concretely for low and medium densities the gran coalition is often formed. Therefore, our results show that larger differences between prices in the two markets leads to larger coalition sizes and that the structure of the coalitions formed is very sensitive to these market conditions.

Finally, observe that as we increase the density of the network, more coalitions of middle size are formed since the size of the maximum coalition decreases with increasing density (e.g. in M1 goes from around a maximum size of 17 to 9) whereas the number of formed coalitions increases (e.g. in M1 goes from around 3 to 6 coalitions). In contrast, low den- 


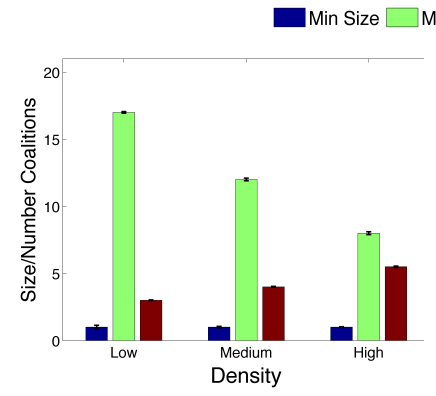

(b) Random Graphs M1.

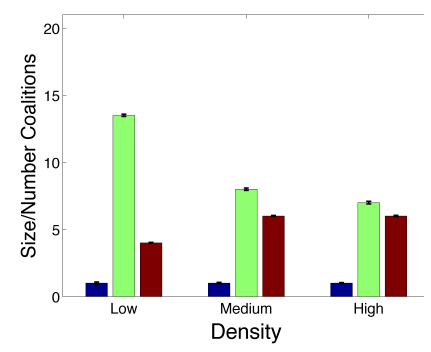

(d) Random Graphs M2.

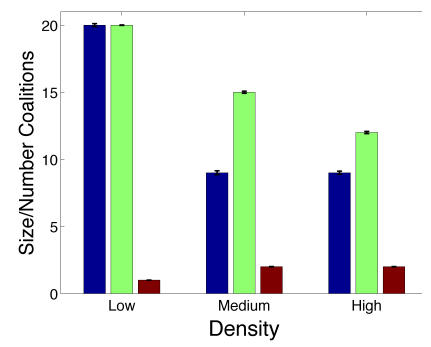

(f) Random Graphs M3.

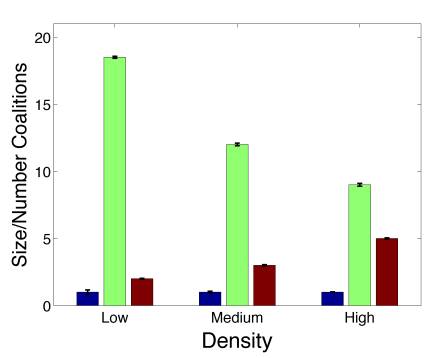

(c) Scale Free M1.

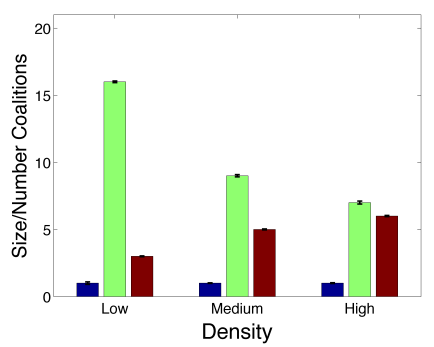

(e) Scale Free M2.

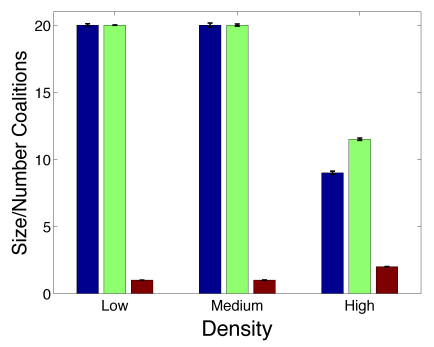

(g) Scale Free M3.

Fig. 3. Graphs showing the minimum/maximum size and number sof coalitions formed on different topologies under market conditions M1 (a)-(b), M2 (c)-(d) and M3 (e)-(f).

sity networks tend to lead to larger coalitions. This trend is observed for all tested market conditions.

\section{CONCLUSIONS AND FUTURE WORK}

In this work we propose a novel energy group buying strategy promoting the formation of customers' coalitions (called Virtual Electricity Consumers or VEC) that can buy energy as aggregates. We model the VEC formation process as a coalitional game using social networking as a tool for consumers to provide member engagement and trust. Moreover, we provide an algorithm (based on linear programming techniques) that identifies the most efficient VEC's and allocates payments to individual members so that formed VECs are stable (i.e., no customer has any incentive to break away). Finally, we defined a metric to evaluate coalitions that takes into account customers' cost for electricity and tested our model on a real dataset of customers' demand, varying crucial parameters such as topology and density of the social network as well as market conditions.

Results show that: i) the density of the social network

affects the stability of the economical agreement among consumers and in dense networks such stability often does not exist; ii) the difference between the price of electricity in the forward and day-ahead markets results in higher consumer gains. This suggests that the difference between the electricity price in the forward and day-ahead markets can be an effective parameter to encourage the formation of bigger coalitions that can buy more electrical energy on the forward market resulting in a more predictable demand for the whole system.

\section{REFERENCES}

[1] Federal Energy Regulatory Commission, "Assessment of demand response and advanced metering," Tech. Rep., FERC Report, 2006.

[2] M.M. Abdullah and B. Dwolatzky, "Demand-side energy management performed using direct feedback via mobile systems: Enables utilities to deploy consumer based demand response programs," in EnergyCon, 2010, pp. 172 -177.

[3] "Tradewind, further developing europe's power market for large scale integration of wind power. report d. 4.1.," 2007, http://www.trade-wind.eu/fileadmin/documents/ publications/D4.1_Summary_report_of_market_rules.pdf.

[4] "Vademecum of the Italian Power Exchange," Gestore dei mercati energetici S. p. A., 2009, http: //www.mercatoelettrico.org/En/MenuBiblioteca/ documenti/20091112VademecumofIpex.pdf.

[5] M. J. Osborne and A. Rubinstein, A Course in Game Theory, MIT Press, Cambridge MA, USA, 1994.

[6] G. Gutin, A. Johnstone, J. Reddington, E. Scott, A. Soleimanfallah, and A. Yeo, "An algorithm for finding connected convex subgraphs of an acyclic digraph," in Algorithms and Complexity. 2008, College Publications.

[7] Rina Dechter, Constraint processing, Elsevier Morgan Kaufmann, 2003.

[8] Talal Rahwan, Algorithms for Coalition Formation in MultiAgent Systems, Ph.D. thesis, University of Southampton, August 2007.

[9] Tomasz Michalak, Jacek Sroka, Talal Rahwan, Michael Wooldridge, Peter Mcburney, and Nicholas Jennings, "A distributed algorithm for anytime coalition structure generation," in AAMAS, 2010, pp. 1007-1014.

[10] Talal Rahwan and N. R. Jennings, "An improved dynamic programming algorithm for coalition structure generation," in $A A$ MAS, 2008, pp. 1417-1420.

[11] M.E.J. Newman, Networks: An Introduction, Oxford University Press, 2010.

[12] G. Demange, "On Group Stability in Hierarchies and Networks," Journal of Political Economy, vol. 112, no. 4, pp. 754-778, 2004. 